\title{
DOF ANALYSIS IN A TWO-LAYERED HETEROGENEOUS WIRELESS INTERFERENCE NETWORK
}

\author{
Meghana Bande, Venugopal V. Veeravalli \\ ECE Department and CSL \\ University of Illinois at Urbana-Champaign \\ Email: \{mbande2,vvv\}@illinois.edu
}

\author{
Antti Tölli, Markku Juntti \\ Centre for Wireless Communication, \\ University of Oulu, Finland \\ Email: \{antti.tolli, markku.juntti\}@ee.oulu.fi
}

\begin{abstract}
Degrees of freedom (DoF) is studied in the downlink of a heterogenous wireless network modeled as a two-layered interference network. The first layer of the interference network is the backhaul layer between macro base stations (MBs) and small cell base stations (SBs), which is modeled as a Wyner type linear network. The second layer is the transmission layer between SBs and mobile terminals (MTs), which is modeled as a linear Wyner $L_{T}$ network. The SBs are assumed to be halfduplex, thus restricting the per user degrees of freedom (puDoF) in the system to $1 / 2$. It is established that the optimal puDoF of $1 / 2$ can be achieved in the linear network with sufficient number of antennas using only interference avoidance schemes. For the case of higher connectivity in the transmission layer, it is shown that the optimal puDoF is achieved by sending an appropriate linear combination to the SB to zero-force interference at the intended user. These results are also extended to a more realistic hexagonal cellular model.

Index Terms-interference management, coordinated multipoint transmission (CoMP), half-duplex relays, interference avoidance, heterogeneous networks.
\end{abstract}

\section{INTRODUCTION}

To meet the increasing demand for mobile traffic, heterogeneous networks are envisioned to be a key component of future cellular networks [1]. Heterogeneous networks enable flexible and low-cost deployments and provide a uniform broadband experience to users anywhere in the network [2]. Managing interference in heterogeneous networks is crucial in order to achieve higher data rates for users. We consider the downlink of a heterogeneous network with $K_{B}$ macro base stations (MBs), $K$ small cell base stations (SBs) and $K$ mobile terminals (MTs) with $K=S \times K_{B}$ for an appropriate $S$. It is assumed that the MBs and the SBs operate on the same frequency band.

The SBs act as relays between MBs and MTs. We consider a linear interference model for both the backhaul and the transmission layer. The channel between the macro and the SBs is modeled as a Wyner type network while the channel between SBs and MTs is modeled as a Wyner $L_{T}$ model. The dependence of DoF in this network on several factors such as the number of antennas at the MB, interference in the backhaul and transmission layers is investigated in this work. These insights are applied to a practical model with the transmission layer modeled as a hexagonal cellular network.

The DoF in locally connected linear networks was studied in [3], [4], [5] using cooperation under maximum transmit set size cooperation constraints. We use insights from these works to characterize the DoF in the transmission layer. In the schemes of [3]-[6], the messages of multiple users are available at some of the transmitters. This requires multiple time-slots in the backhaul layer in our setting. The key observation here is that the SBs use the knowledge of multiple messages to null the interference at the intended MT. Hence it is sufficient if the MBs send linear combinations of the messages to the SBs. This would require that at each MB, the channel between SBs and the corresponding MTs is known.

A two-layered interference network modeled as a $K \times K \times K$ relay channel with each layer as a $K$-user interference channel with full connectivity was considered in [8]. Using alignednetwork-diagonalization, the maximum sum-DoF of $K$ was achieved. The sum-DoF was studied for the case of $K=2$ i.e., $2 \times 2 \times 2$ in [9] under restriction to linear schemes, and was shown to be $4 / 3$. In contrast to these schemes, we consider a broadcast channel in the first layer and local connectivity in both layers. We also restrict ourselves to more practical zeroforcing schemes. To the best of our knowledge, there has been no prior work on a DoF analysis for a two-layered network of the kind we consider here.

\section{System Model AND Notation}

We consider a heterogeneous wireless network with MBs, SBs and MTs. It is assumed that MBs do not directly serve MTs and do not cause interference at MTs. We assume that the SBs act as half-duplex relays between the MBs and the MTs. There are two layers in this network, the backhaul layer between MBs and SBs and the transmission layer between SBs and MTs.

\section{A. Transmission Layer}

Consider the transmission layer with $K$ SBs and MTs. Let $\mathcal{K}$ denote the set $\{1, \ldots, K\}$. Each SB is equipped with a single 
antenna. In the transmission layer, the channel gain between SB $j, \forall j \in \mathcal{K}$ and MT $i, \forall i \in \mathcal{K}$ is denoted by $h_{j i}^{\mathrm{Tx}}$. At each MT $i$, the received signal $y_{i}^{\text {Tx }}$ is given by

$$
y_{i}^{\mathrm{Tx}}(t)=h_{i i}^{\mathrm{Tx}}(t) x_{i}^{\mathrm{Tx}}(t)+\sum_{j \in \mathcal{I}_{i}} h_{j i}^{\mathrm{Tx}}(t) x_{j}^{\mathrm{Tx}}(t)+z_{i}^{\mathrm{Tx}}(t),
$$

where $t$ denotes the time-slot, $x_{j}^{\mathrm{Tx}}(t)$ denotes the signal transmitted by SB $j$ under an average transmit power constraint, $z_{i}^{\mathrm{Tx}}(t)$ denotes the additive white Gaussian noise at MT $i$, $h_{j i}^{\mathrm{Tx}}(t)$ denotes the channel gain coefficient from SB $j$ to MT $i$, and $\mathcal{I}_{i}$ denotes the set of interferers at MT $i$.

The cellular model presented by Wyner [10] was extended in [5] to a locally connected linear interference network with connectivity parameter $L_{T}$. The transmission layer is assumed to be a local Wyner $L_{T}$ model with $K$ users. The cells are located on an infinite linear equi-spaced grid and each transmitter is associated with a single user. Here $L_{T}$ denotes the number of dominant interferers per user, where each user observes interference from $\left\lceil\frac{L_{T}}{2}\right\rceil$ preceding and $\left\lfloor\frac{L_{T}}{2}\right\rfloor$ succeeding transmitters. The Wyner $L_{T}$ model is illustrated in Figure 2 and the channel coefficients are given by

$h_{j i}^{\mathrm{Tx}}(t) \neq 0$ iff $i \in\left\{j-\left\lfloor\frac{L_{T}}{2}\right\rfloor, \ldots, j-1, j, j+1, \ldots, j+\left\lceil\frac{L_{T}}{2}\right\rceil\right\}$.

\section{B. Backhaul Layer}

The backhaul layer is assumed to be a linear model with connectivity $L_{B}$. Let $\mathcal{S}_{i}$ denote the set of $S$ consecutive SBs where $\mathcal{S}_{i}=\{(i-1) S+1, \ldots,(i) S\}$. Each MB $i$ is associated with a set $\mathcal{A}_{i}$ of $S+L_{B}$ consecutive SBs where $\mathcal{A}_{i}=\mathcal{S}_{i-1}\left(\left\{S-\left\lfloor\frac{L_{B}}{2}\right\rfloor, \ldots, S\right\}\right) \cup \mathcal{S}_{i} \cup \mathcal{S}_{i+1}\left(\left\{1, \ldots,\left\lceil\frac{L_{B}}{2}\right\rceil\right\}\right)$. Transmission from MB $i$ to any SB in $\mathcal{S}_{i}$ causes interference at $\left\lfloor\frac{L_{B}}{2}\right\rfloor$ SBs above and at $\left\lceil\frac{L_{B}}{2}\right\rceil$ SBs below the set $\mathcal{S}_{i}$. Let $N$ denote the number of antennas at each MB.

The channel vector between MB $i$ and SB $j$ at time-slot $t$ is denoted by $\mathbf{h}_{i, j}^{B}(t)$. The channel model for backhaul layer is given by $\mathbf{h}_{i, j}^{B}(t) \neq 0$ iff $j \in \mathcal{A}_{i}$. Let the channel gain matrix corresponding to $\mathrm{MB} i, \mathbf{H}_{i}^{B}(t) \in \mathbb{C}^{(N) \times\left(S+L_{B}\right)}=$ $\left[\mathbf{h}_{i, \mathcal{S}_{i-1}\left(S-\left\lfloor\frac{L_{B}}{2}\right\rfloor\right)}^{B}(t), \ldots, \mathbf{h}_{i, \mathcal{S}_{i}(S)}^{B}(t), \ldots, \mathbf{h}_{i, \mathcal{S}_{i+1}\left(\left\lceil\frac{L_{B}}{2}\right\rceil\right)}^{B}(t)\right]$ in the backhaul layer where the $j$ th column corresponds to the channel coefficients from MB $i$ to SB $j$. Also let $\mathbf{x}_{i}^{B}(t) \in \mathbb{C}^{N \times 1}$ to be the transmitted signal vector from MB $i$ and $z_{k}^{B}(t)$ denotes the additive white Gaussian noise at SB $k$. The received signal at $k$ th SB served by MB $i$ is given by,

$$
y_{k}^{B}(t)=\left(\mathbf{h}_{i, k}^{B}(t)\right)^{T} \mathbf{x}_{i}^{B}(t)+\sum_{j \neq i}\left(\mathbf{h}_{j, k}^{B}(t)\right)^{T} \mathbf{x}_{j}^{B}(t)+z_{k}^{B}(t) .
$$

Let $\mathcal{R}_{i}(t) \subseteq \mathcal{A}_{i}$ denote the set of SBs receiving messages from MB $i$ in a particular time-slot $t$.

Local channel state information is assumed to be available at MBs and SBs. All channel coefficients that are not identically zero are assumed to be drawn independently from a continuous joint distribution. The system model is illustrated in Figure 1.

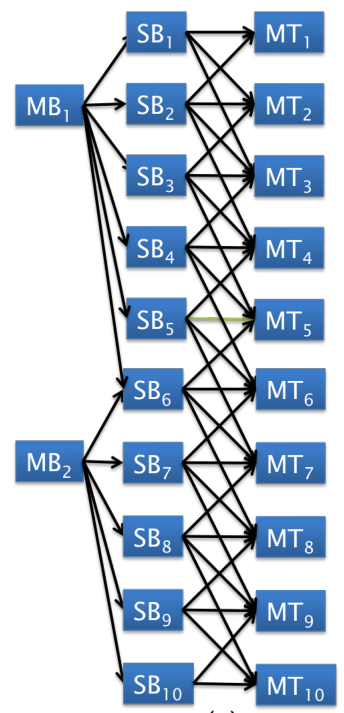

(a)

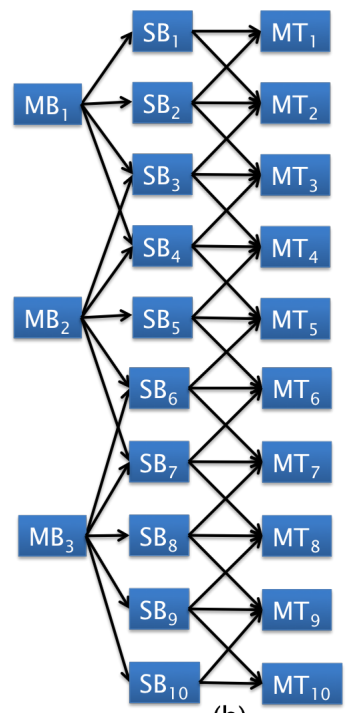

(b)
Fig. 1: Two-layered network with: (a) $S=5$ and $L_{B}=1$ in the backhaul layer, and $L_{T}=3$ in the transmission layer; and (b) $S=3$ and $L_{B}=2$ in the backhaul layer, and $L_{T}=2$ in the transmission layer.

\section{Capacity and Degrees of Freedom}

Let $P$ be the average transmit power constraint at each SB and the transmit power per antenna at a MB. Let $\mathcal{W}_{i}$ denote the alphabet for $W_{i}$, where $W_{i}$ denotes the message for MT $i$. The rates $R_{i}(P)=\frac{\log \left|\mathcal{W}_{i}\right|}{n}$ are achievable iff the error probabilities of all messages can simultaneously be arbitrarily small for large $n$, using an interference management scheme. The degree of freedom (DoF) $d_{i}, \forall i \in \mathcal{K}$ is defined as

$$
d_{i}=\lim _{P \rightarrow \infty} \frac{R_{i}(P)}{\log P} .
$$

DoF is the number of interference free sessions in a multi-user channel at asymptotically high signal-to-noise ratio (SNR). The maximum achievable sum DoF $\eta(K)$ in a channel with $K$ users (MTs) is defined as $\eta(K)=\max _{\mathcal{D}} \sum_{i \in \mathcal{K}} d_{i}$. where $\mathcal{D}$ denotes the closure of the set of all achievable DoF tuples. Then the maximum achievable per user DoF (puDoF) is defined as

$$
\tau=\lim _{K \rightarrow \infty} \frac{\eta(K)}{K} .
$$

\section{DoF AnALYSIS FOR $L_{T}=\{1,2\}$}

In this section, we consider the case where the connectivity in the transmission layer $L_{T} \leq 2$ while $L_{B}=1$. We present upper bounds on the achievable puDoF which hold for general $L_{T}$ and $L_{B}$. Similar achievability schemes can be used for higher values of $L_{T}$ and $L_{B}$.

Note that at any MB $i, N_{1}+1$ antennas are sufficient in order to send messages to $N_{1}$ SBs $\left(\left|\mathcal{R}_{i}\right|=N_{1}\right)$ and to null the interference at $\mathrm{SB} z$. Let $\mathbf{X} \in \mathbb{C}^{N_{1}+1}$ denote the transmitted signal vector at $\mathrm{MB} i, \mathbf{H}$ denote $\left[\mathbf{H}_{i, \mathcal{R}_{i}}^{B}, \mathbf{h}_{i, z}^{B}\right]$, 
and $\mathbf{W} \in \mathbb{C}^{N_{1}+1}$ denote the vector containing the intended messages to $\mathcal{R}_{i}$ appended with zero at the end. Then we have $\mathbf{H X}^{T}=\mathbf{W}^{T}$. From our assumptions, $\mathbf{H}$ is full rank almost surely and a solution for $\mathbf{X}$ is obtained.

Theorem 1: The following bounds hold for $\tau$, when $L_{B}=1$, $L_{T} \in\{1,2\}$,

$$
\begin{gathered}
\tau \geq\left\{\begin{array}{cl}
\frac{N}{S} \quad & \text { for } N<S / 2 \\
\frac{1}{2}\left(1 \frac{1}{S}\right) & \text { for } N=S / 2 \text { for } S \text { even } \\
\frac{1}{2} \quad & \text { for } N>S / 2 \\
\tau \leq \min \left(\frac{N}{S}, \frac{1}{2}\right) .
\end{array}\right.
\end{gathered}
$$

Proof: We present the achievable scheme in order to show the bounds (4). The converse (5) is omitted due to space constraints and can be found in the full version of this paper [12]. In the transmission layer for Wyner model with $L_{T} \in\{1,2\}$, by deactivating alternate transceiver pairs, the remaining messages can be sent interference free as shown in Figure 2. Thus, a puDoF of $1 / 2$ is achieved if the corresponding messages are available at the active SBs.

Case 1: $N>\frac{S}{2}$.

A) When $S$ is odd, our achievable scheme uses only $\frac{S+1}{2}$ antennas at an MB. Consider the following message assignment for each time-slot $t$ where $t$ is odd.

$$
\mathcal{R}_{i}(t)=\begin{array}{ll}
\mathcal{S}_{i}(\{1,3, \ldots, S\}) & \text { for } i \text { odd } \\
\mathcal{S}_{i}(\{2,4, \ldots, S-1\}) & \text { for } i \text { even. }
\end{array}
$$

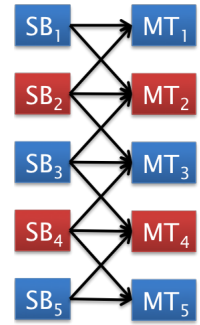

Fig. 2: Scheme achieving puDoF $=1 / 2$ in the transmission layer with $L_{T}=2$. The red boxes indicate deactivated transceivers.

When $i$ is even, $\mathrm{SB} \mathcal{S}_{i}(1)$ is not active in this time-slot. Only when $i$ is odd, $\mathcal{S}_{i}(1)$ observes interference from the transmissions of $\mathrm{MB} i-1$. MB $i-1$ needs $\frac{S-1}{2}$ antennas for sending messages and one antenna for nulling the interference at $\mathrm{SB} \mathcal{S}_{i}(1)$. Thus at the end of each odd time-slot, messages are available at alternate $\mathrm{SBs}$ and a puDoF of $1 / 2$ is achieved. The assignment is reversed when $t$ is even and the achievability follows similarly.

B) The proof for $S$ is even is omitted due to space constraints and can be found in the full version of the paper [12].

Case 2: $N<\frac{S}{2}$. In this case, $S \geq 2 N+2$ or $S \geq 2 N+1$ for even and odd respectively. Hence in each cluster, two disjoint sets of $N$ SBs are served in consecutive time-slots while the first SB of the cluster is inactive. Consider the following message assignment for each time-slot $t$ when $t$ is odd.

$$
\mathcal{R}_{i}(t)=\begin{array}{ll}
\mathcal{S}_{i}(\{3,5, \ldots, 2 N+1\}) & \text { for } i \text { odd } \\
\mathcal{S}_{i}(\{2,4, \ldots, 2 N\}) & \text { for } i \text { even. }
\end{array}
$$

This assignment is reversed when $t$ is even. The first SB in each cluster is not served at all and hence there is no interference in the backhaul layer. In each time-slot, $N$ messages among every $S$ users are sent interference-free, achieving a puDoF of $\frac{N}{S}$.

Case 3: $N=\frac{S}{2}$. The proof is omitted due to space constraints and can be found in the full version of our paper [12].

The upper bounds in Equation (5) hold in general for all possible achievable schemes, but the maximum puDoF can be achieved by simple interference avoidance schemes except for the case $N=\frac{S}{2}$ when $L_{T} \in\{1,2\}$. The achievable schemes are illustrated in Figure 3 . The green and orange arrows indicate the transmissions in consecutive time-slots and blue, the nulling beam. Even for a general $L_{B}$, by employing a sufficiently large number of antennas $N \geq\left\lceil\frac{S}{2}\right\rceil+L_{B}$ at the macro base stations, the interference in backhaul layer can be eliminated and a puDoF of $1 / 2$ can be achieved.

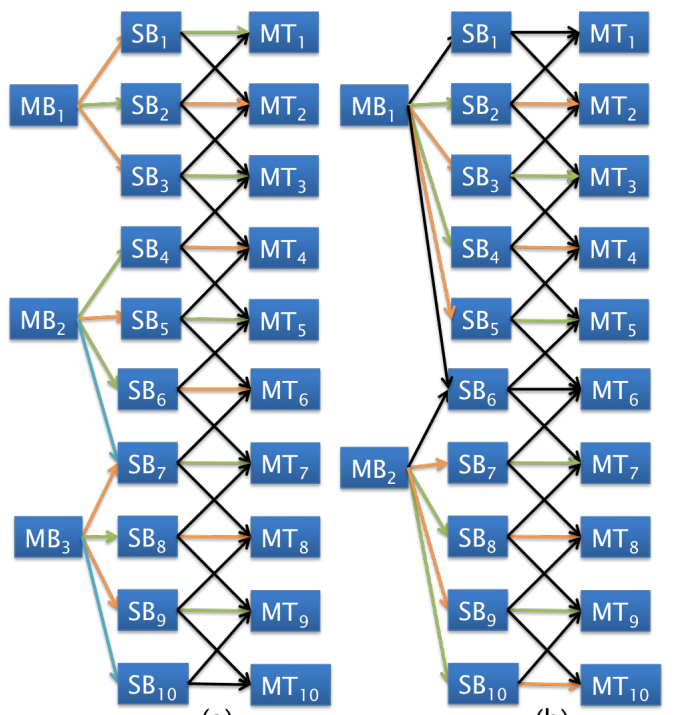

(b)

Fig. 3: Achievable schemes for the network with $L_{B}=1$ and $L_{T}=2$ : (a) puDoF $=1 / 2$ with $S=3$ and $N=2$; and (b) $\mathrm{puDoF}=2 / 5$ with $S=5$ and $N=2$.

\section{AChievable schemes fOR General $L_{T}$}

The optimal puDoF for a given number of antennas cannot be achieved for higher values of $L_{T}$ using only interference avoidance schemes without the use of cooperation. For example, when $L_{T}=3$, with restriction to only $\mathrm{ZF}$ schemes without cooperation at the SBs, we have $\tau \leq \frac{2}{5}$ in the 
transmission layer even for a large $N$ from [5]. We consider cooperation among the SBs and show that the optimal puDoF can be achieved for $L_{T} \in\{3,4\}$ using only interference avoidance schemes. For cooperation, multiple messages need to be available at SBs for transmission in a particular timeslot. This requires multiple time-slots for transmission by the MBs in the backhaul layer which leads to ineffective use of resources. The SBs use the knowledge of messages available only for zero-forcing, and, thus, it suffices to have a linear combination of messages at the SBs. This would require only one time-slot for transmission in the backhaul layer. However, this would require that at each MB, the channel between SBs and the corresponding MTs is known. While this might require a large amount of CSI to be present at each MB, this would be justified if the coherence time is large enough.

Remark 1: In the Wyner $L_{T}$ model, if groups of $A$ SBMT pairs are separated by $F$ where $F \geq\left\lceil\frac{L_{T}}{2}\right\rceil$ deactivated pairs, then there is no interference between the groups. If all $A$ messages are sent such that the interference at each MT is zero-forced, a puDoF of $A /(F+A)$ is achieved if the messages are available at the SBs.

Theorem 2: When $L_{B}=1$ and $\left\lfloor\frac{S}{2}\right\rfloor \geq\left\lceil\frac{L_{T}}{2}\right\rceil$, equations (4) and (5) hold for $\tau$.

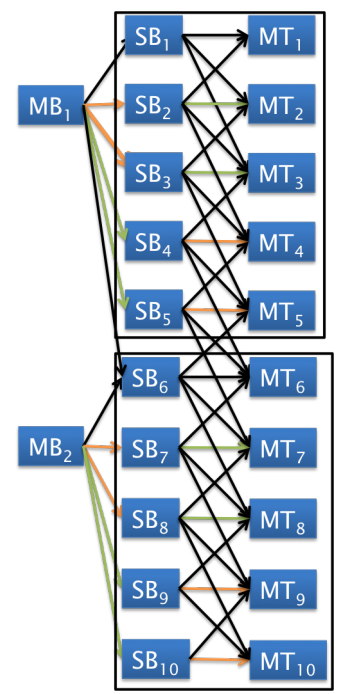

(a)

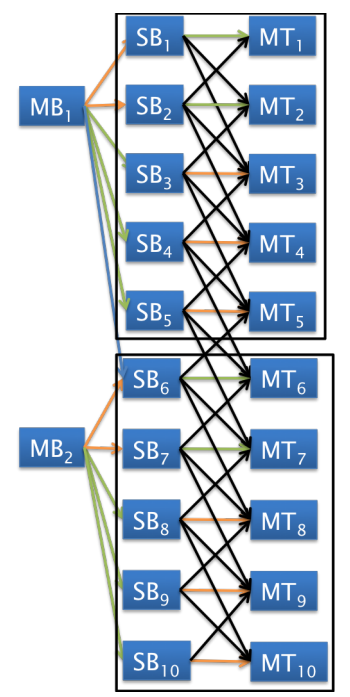

(b)
Fig. 4: Achievable schemes for the network with $L_{B}=1$, $L_{T}=3$ and $S=5$. In (a), $N=2, N<S / 2$ and $\mathrm{puDoF}=$ $2 / 5$ is achieved. In (b), $N=3, N>S / 2$ and $\mathrm{puDoF}=1 / 2$ is achieved.

Proof:

A) $N>\frac{S}{2}$ is equivalent to $N \geq\left\lfloor\frac{S}{2}\right\rfloor+1$. For all $i$, let

$$
\mathcal{R}_{i}(t)=\begin{array}{ll}
\mathcal{S}_{i}\left(\left\{1, \ldots,\left\lfloor\frac{S}{2}\right\rfloor\right\}\right) & \text { for } t \text { odd } \\
\mathcal{S}_{i}\left(\left\{\left\lfloor\frac{S}{2}\right\rfloor+1, \ldots, S\right\}\right) & \text { for } t \text { even. }
\end{array}
$$

In even and odd time-slots, $\left\lfloor\frac{S}{2}\right\rfloor+1$ and $\left\lfloor\frac{S}{2}\right\rfloor$ antennas respectively at each MB $i$ are used to send linear combinations to the SBs and in an odd time-slot one antenna is used to ZF interference at $\mathcal{S}_{i+1}(1)$. From remark 1, it follows that the puDoF is $1 / 2$.

B) $N<\frac{S}{2}$ is same as $N \leq\left\lceil\frac{S}{2}\right\rceil-1$. For all $i$, let

$$
\mathcal{R}_{i}(t)=\begin{array}{ll}
\mathcal{S}_{i}(\{2, \ldots, N+1\}) & \text { for } t \text { odd } \\
\mathcal{S}_{i}\left(\left\{\left\lceil\frac{S}{2}\right\rceil+1, \ldots,\left\lceil\frac{S}{2}\right\rceil+1+N\right\}\right) & \text { for } t \text { even. }
\end{array}
$$

In each time-slot, $N$ antennas at each $\mathrm{MB} i$ send linear combinations to the SBs. The first SB in each cluster is always inactive. The first MT in each cluster is served by an appropriate SB in the cluster to maintain fairness. Each group of $N$ SBs is separated by $S-N$ SBs and hence from remark $1, \mathrm{puDoF}$ is $N / S$.

C) $N=\frac{S}{2}$. The proof is omitted due to space constraints and can be found in the full version of the paper [12].

The achievable schemes are illustrated in Figure 4. We note that for a general $L_{B}$, a puDoF of $1 / 2$ can be achieved by employing a sufficient number of antennas $\left(N \geq\left\lfloor\frac{S}{2}\right\rfloor+L_{B}\right)$.

\section{Cellular model}

We have extended this work to include the more realistic hexagonal sectored cellular model (e.g., used in [11]) for the transmission layer. We assume only local interference and no intra-cell interference in the transmission layer. For the backhaul layer, each $\mathrm{MB}$ is associated with a $3 \times 3$ block of SBs and transmissions in each block cause interference at only the neighboring SBs.

Theorem 3: With $N \geq 13$, a puDoF of $1 / 2$ can be achieved using only zero-forcing schemes.

The proof and additional details about the system model can be found in [12]. We anticipate that having a large number of antennas at the MBs will be realistic in future generation wireless systems with the emergence of massive MIMO [7].

\section{CONCLUSions}

In this paper we analyzed the DoF for a linear Wyner type model for a two-tier heterogeneous wireless network. The system model, while being somewhat simplistic, captures important features of such networks, and leads to new insights for designing more realistic cellular networks. For the linear two-tier heterogeneous wireless network, we showed that with sufficient number of antennas, a puDoF of $1 / 2$ can be achieved using only zero-forcing schemes by sending linear combinations from MBs to their corresponding SBs so that the interference at each MT is zero-forced. These insights apply to a more realistic hexagonal cellular network where a puDoF of $1 / 2$ can be achieved with a sufficient number of antennas using only interference avoidance schemes.

\section{ACKNOWLEDGMENT}

This research was supported in part by the US NSF WiFiUS program under grant CNS 14-57168, through the University of Illinois at Urbana-Champaign, and by the Academy of Finland under grant 284801. 


\section{REFERENCES}

[1] C. X. Wang et al., "Cellular architecture and key technologies for 5G wireless communication networks," in IEEE Communications Magazine, vol. 52, no. 2, pp. 122-130, February 2014.

[2] A. Khandekar, N. Bhushan, Tingfang Ji, V. Vanghi, "LTE-Advanced: Heterogeneous networks," in Wireless Conference (EW), 2010 European, pp. 978-982, 12-15 April 2010.

[3] A. Lapidoth, S. Shamai (Shitz) and M. A. Wigger, "A linear interference network with local side-information," in Proc. IEEE International Symposium on Information Theory (ISIT), pp. 2201-2205, Nice, Jun. 2007.

[4] S. Shamai (Shitz) and M. A. Wigger, "Rate-limited transmittercooperation in Wyner's asymmetric interference network," in Proc. IEEE International Symposium on Information Theory (ISIT), pp. 425-429, Saint Petersburg, Aug. 2011.

[5] A. El Gamal, V. S. Annapureddy, and V. V. Veeravalli, "Interference channels with coordinated multi-point transmission: Degrees of freedom, message assignment, and fractional reuse." IEEE Trans. Inf. Theory, vol. 60, no. 6, pp. 3483-3498, Jun. 2014

[6] A. El Gamal, V. V. Veeravalli, "Dynamic Interference Management," Asilomar Conference on Signals, Systems and Computers, pp.1902-1906, Nov. 2013.

[7] E. G. Larsson, O. Edfors, F. Tufvesson and T. L. Marzetta, "Massive MIMO for next generation wireless systems," in IEEE Communications Magazine, vol. 52, no. 2, pp. 186-195, February 2014.

[8] I. Shomorony and A. S. Avestimehr, "Degrees of freedom of two-hop wireless networks: Everyone gets the entire cake," IEEE Trans. Inf. Theory, vol. 60, no. 5, pp. 24172431, May 2014.

[9] Issa, I.; Fong, S.L.; Avestimehr, A.S., "Two-Hop Interference Channels: Impact of Linear Schemes," IEEE Trans. Inf. Theory, vol.61, no.10, pp.5463-5489, Oct. 2015.

[10] A. D. Wyner, "Shannon-theoretic approach to a Gaussian cellular multiaccess channel," IEEE Trans. Inf. Theory, vol. 40, no. 6, pp. 17131727, Nov. 1994.

[11] M. Bande, A. El Gamal and V. V. Veeravalli, "Flexible Backhaul Design with Cooperative Transmission in Cellular Interference Networks," International Symposium on Information Theory (ISIT). Jul. 2015

[12] M. Bande, V. V. Veeravalli, A. Tolli and M. Juntti "DoF Analysis in a Two-Layered Heterogeneous Wireless Interference Network," Arxiv preprint, http://arxiv.org/pdf/1609.02117v1.pdf. 